Kettering University Digital Commons @ Kettering University

$5-29-2014$

Fate of Sessile Droplet Chemical Agents in Environmental Substrates in the Presence of Physiochemical Processes

Homayun K. Navaz

A. L. Dang

Theresa Atkinson

Ali Zand

A. Nowakowski

See next page for additional authors

Follow this and additional works at: https://digitalcommons.kettering.edu/mech_eng_conference

Part of the Mechanical Engineering Commons 
Authors

Homayun K. Navaz, A. L. Dang, Theresa Atkinson, Ali Zand, A. Nowakowski, and K. Kamensky 


\section{Fate of sessile droplet chemical agents in environmental substrates in the presence of physiochemical processes}

Navaz, H., Dang, A., Atkinson, T., Zand, A., Nowakowski, A., et al.

H. K. Navaz, A. L. Dang, T. Atkinson, A. Zand, A. Nowakowski, K. Kamensky, "Fate of sessile droplet chemical agents in environmental substrates in the presence of physiochemical processes," Proc. SPIE 9073, Chemical, Biological, Radiological, Nuclear, and Explosives (CBRNE) Sensing XV, 90730A (29 May 2014); doi: 10.1117/12.2049595

SPIE. Event: SPIE Defense + Security, 2014, Baltimore, Maryland, United States 


\title{
Fate of sessile droplet chemical agents in environmental substrates in the presence of physiochemical processes
}

\author{
H. K. Navaz, A. L. Dang, T. Atkinson, A. Zand, A. Nowakowski, K. Kamensky
}

\begin{abstract}
A general-purpose multi-phase and multi-component computer model capable of solving the complex problems encountered in the agent substrate interaction is developed. The model solves the transient and time-accurate mass and momentum governing equations in a three dimensional space. The provisions for considering all the inter-phase activities (solidification, evaporation, condensation, etc.) are included in the model. The chemical reactions among all phases are allowed and the products of the existing chemical reactions in all three phases are possible. The impact of chemical reaction products on the transport properties in porous media such as porosity, capillary pressure, and permeability is considered. Numerous validations for simulants, agents, and pesticides with laboratory and open air data are presented. Results for chemical reactions in the presence of pre-existing water in porous materials such as moisture, or separated agent and water droplets on porous substrates are presented. The model will greatly enhance the capabilities in predicting the level of threat after any chemical such as Toxic Industrial Chemicals (TICs) and Toxic Industrial Materials (TIMs) release on environmental substrates. The model's generality makes it suitable for both defense and pharmaceutical applications.
\end{abstract}

\section{INTRODUCTION}

There are numerous engineering applications which involve the spread of a fluid phase within a porous medium. Underground water movement, oil recovery, fracking, pharmaceuticals, drug delivery systems, chemical agent defense, polymer filling, printing, study of membranes and multi-layer materials, and fuel cell are several industries that are related to porous media flow. Researchers in the past have solved the porous media flow by continuum or discrete methods. This volume of work has been addressed by Markicevic et al [1-14]. The flow through porous materials becomes more complicated when evaporation on the surface and within the pores is also present [15-16]. Furthermore, sometimes a chemical enters a reaction with other pre-existing chemicals or the substrate in the porous medium. This is generally encountered in applications like pharmaceuticals, drug delivery process, designing protective gears, and chemical agent interaction with environmental substrates. In the latter application chemical agents (or pesticides) are generally released after an explosion or atomization process leading to dissemination in droplet form that initially resides on a surface, i.e., sessile droplets.

The spread of chemical warfare agents (CWA's), TICs, TIMs, and pesticides in the environment pose a great threat to warfighters and human lives and health. This threat becomes more serious if their release takes place with malicious intentions. The field commanders and/or first responders have to make quick and often difficult decisions that have direct impacts on the lives of the soldiers and the citizens. It will be preferred that these decisions to be based on scientific analysis and data regarding the spread and distribution of these chemicals into the environmental substrates or atmosphere. Understanding the interaction of a liquid with a porous material with or without previously existing chemicals inside the pores is critical for determining the fate of toxic chemicals. This problem has environmental, defense, and homeland security implications where assessing the contamination level and the possible need for decontamination is critical to evaluating and mitigating the threat. When a chemical agent is disseminated onto environmental substrates several processes occur simultaneously. These processes are surface evaporation (as long as a sessile droplet is visible on the surface), capillary transport through the substrate, evaporation inside the pores, chemical reaction with the substrate and/or other pre-existing chemicals inside the pores, especially moisture and water, and adsorption/desorption processes.

To the best of our knowledge, there is no existing general-purpose computer model that couples all existing physiochemical processes by solving fully coupled conservation equations in 3-dimention. The need and the lack of a credible computer model that can be used as a workhorse (so called production code) prompted the development of a

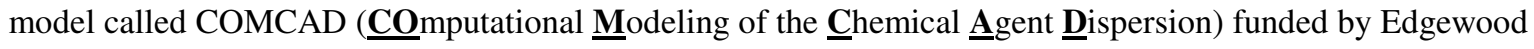


Chemical and Biological Center (ECBC) [16]. The COMCAD model was enhanced by a new chemical reaction model and addition of contact dynamics and mass transfer funded by Defense Threat Reduction Agency (DTRA) [17]. The resulting model can be used to predict the threat of hazardous materials released onto environmental substrates with or without a contacting surface. The new model is called the MOCHA (Modeling $\underline{\mathbf{O}}$ the $\underline{\mathbf{C H}} \mathrm{emical}$

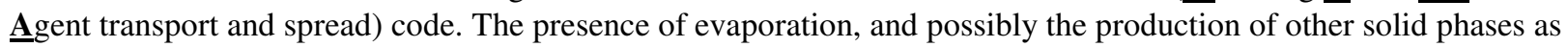
a result of existing chemical reactions transitioned the MOCHA code into a multi-phase formulation making it a general-purpose computer program for porous media. We will briefly discuss the governing equations and numerical solution followed by a variety of test cases to further demonstrate the capabilities of the model.

\section{MODEL DESCRIPTION}

The governing equations for each phase are derived in Reference [17] as shown in Equations (1). Note that the energy equation is written for a lumped system, i.e. all phases have the same temperature. The equations account for evaporation, phase change, and chemical reaction.

\section{Solid Phase}

$\frac{\partial \rho_{s k} \phi_{s}}{\partial t}=-\dot{\omega}_{s k}^{\text {Re action }}-\dot{\omega}_{s k}^{\text {Adsorption }}$

$\phi_{s}=1-\phi$

\section{Liquid Phase}

$\frac{\partial\left(\phi \rho_{\ell i} s_{\ell i}\right)}{\partial t}+\nabla \bullet\left(\phi \rho_{\ell i} s_{\ell i} \vec{V}_{\ell i}\right)=\left(-\dot{\rho}_{\ell i}^{\text {Secondary Evaporation }}-\dot{\rho}_{\ell i}^{\text {Surface Evaporation }}-\dot{\omega}_{\ell i}^{\text {Re action }}-\dot{\omega}_{\ell i}^{\text {Adsorption }}\right)$

gas Phase

$\frac{\partial\left(\phi \rho_{g j} s_{g}\right)}{\partial t}+\nabla \bullet\left(\phi \rho_{g j} s_{g} \vec{V}_{g j}\right)=\frac{\partial}{\partial x}\left[\rho_{g} D_{j-m i x} \frac{\partial C_{j}}{\partial x}\right]+\frac{\partial}{\partial y}\left[\rho_{g} D_{j-m i x} \frac{\partial C_{j}}{\partial y}\right]+$

$\frac{\partial}{\partial z}\left[\rho_{g} D_{j-m i x} \frac{\partial C_{j}}{\partial z}\right]+\dot{\rho}_{\rho_{f i}}^{\text {Secondary Evaporation }}-\dot{\omega}_{g j}^{\text {Re action }}-\dot{\omega}_{g j}^{\text {Adsorption }}$

Momentum

Liquid Phase: $\quad \vec{V}_{\ell i}=-\frac{K k_{\ell i}}{\mu_{\ell}}\left(\nabla P_{\ell i}-\rho_{\ell i} g s_{\ell i}\right)$,

Gas Phase: $\vec{V}_{g j}=-\frac{K k_{g j}}{\mu_{g}}\left(\nabla P_{g j}-\rho_{g j} g s_{g}\right)-D_{j \rightarrow \text { mixture }} \nabla C_{g j}$,

Energy

$$
\begin{aligned}
& \frac{\partial}{\partial t}\left[H_{s}+H_{\ell}+H_{g}\right]+\nabla \bullet\left(\vec{V}_{\ell} H_{\ell}\right)+\nabla \bullet\left(\vec{V}_{g} H_{g}\right)=-\nabla \bullet\left(\vec{V}_{\ell} \bullet \boldsymbol{\tau}_{\ell}\right)-\nabla \bullet\left(\vec{V}_{g} \bullet \boldsymbol{\tau}_{g}\right)+\dot{\mathbf{q}} \\
& +\left[-\rho_{\ell} v_{\ell} g-\rho_{g} v_{g} g\right]+\sum_{i=1}^{N_{\text {solids }}} \dot{\rho}_{s-\ell i} \Delta h_{s-\ell i}+\sum_{i=1}^{N_{\text {liquids }}} \dot{\rho}_{\ell-g i} \Delta h_{\ell-g i}+\sum_{i=1}^{N_{\text {solids }}} \dot{\rho}_{s-g i} \Delta h_{s-g i}=0
\end{aligned}
$$


Velocities are mass averaged

$$
\begin{gathered}
H_{s}=\rho_{s} c_{p s} \phi_{s} T \quad H_{\ell}=\rho_{\ell} \phi_{\ell}\left(c_{p \ell} T+\frac{V_{\ell}^{2}}{2}\right) \quad H_{g}=\rho_{g} \phi_{g}\left(c_{p g} T+\frac{V_{g}^{2}}{2}\right) \\
V_{\ell}^{2}=\sqrt{u_{\ell}^{2}+v_{\ell}^{2}+w_{\ell}^{2}},
\end{gathered}
$$

where: $s_{g}=1-\sum_{i}^{N(\text { Liquids })} s_{\ell i} \quad$ and $\rho_{g}=\sum_{j=1}^{M \text { (Gases })} \rho_{g j}$

$D_{j-m i x}=$ Effective Diffusion Coefficient of gas species " $j "$ int $o$ the mixture

$C_{j}=$ Mass Fraction $=\frac{\rho_{g j}}{\rho_{g}} \quad P_{\ell i}=P-P_{c}$ (Capillary), $K=$ Saturation permeability, $\mu=$ Viscosity $\rho_{\ell}=$ Density of liquid phase,$\quad s_{\ell}=$ Saturation of liquid

The equations are transformed into computational coordinate system by:

$$
\begin{aligned}
& \xi=\xi(x, y, z) \\
& \eta=\eta(x, y, z) \\
& \zeta=\zeta(x, y, z)
\end{aligned}
$$

With:

$$
\begin{aligned}
& \frac{\partial}{\partial x}=\xi_{x} \frac{\partial}{\partial \xi}+\eta_{x} \frac{\partial}{\partial \eta}+\zeta_{x} \frac{\partial}{\partial \zeta} \\
& \frac{\partial}{\partial y}=\xi_{y} \frac{\partial}{\partial \xi}+\eta_{y} \frac{\partial}{\partial \eta}+\zeta_{y} \frac{\partial}{\partial \zeta} \\
& \frac{\partial}{\partial z}=\xi_{z} \frac{\partial}{\partial \xi}+\eta_{z} \frac{\partial}{\partial \eta}+\zeta_{z} \frac{\partial}{\partial \zeta}
\end{aligned}
$$

Where

$$
\begin{array}{ll}
\xi_{x}=\frac{y_{\eta} z_{\zeta}-y_{\zeta} z_{\eta}}{|\mathbf{n}|}, & \xi_{y}=-\frac{x_{\eta} z_{\zeta}-x_{\zeta} z_{\eta}}{|\mathbf{n}|}, \quad \xi_{z}=-\frac{x_{\eta} y_{\zeta}-x_{\zeta} y_{\eta}}{|\mathbf{n}|} \\
\eta_{x}=-\frac{y_{\xi} z_{\zeta}-y_{\zeta} z_{\xi}}{|\mathbf{n}|}, & \eta_{y}=\frac{x_{\xi} z_{\zeta}-x_{\zeta} z_{\xi}}{|\mathbf{n}|}, \quad \eta_{z}=-\frac{x_{\xi} y_{\zeta}-x_{\zeta} y_{\xi}}{|\mathbf{n}|} \\
\zeta_{x}=\frac{y_{\xi} z_{\eta}-y_{\eta} z_{\xi}}{|\mathbf{n}|}, & \zeta_{y}=-\frac{x_{\xi} z_{\eta}-x_{\eta} z_{\xi}}{|\mathbf{n}|}, \quad \zeta_{z}=\frac{x_{\xi} y_{\eta}-x_{\eta} y_{\xi}}{|\mathbf{n}|}
\end{array}
$$

With the norm of:

$$
|\mathbf{n}|=x_{\xi} y_{\eta} z_{\zeta}-x_{\xi} y_{\zeta} z_{\eta}+x_{\eta} y_{\zeta} z_{\xi}-x_{\eta} y_{\xi} z_{\zeta}+x_{\zeta} y_{\xi} z_{\eta}-x_{\zeta} y_{\eta} z_{\xi}
$$




\section{CHEMISTRY MODEL}

A general chemical reaction can be written in terms of its stoichiometric coefficients $v_{i j}$ and $v_{i j}^{\prime}$ as:

$$
\sum_{i=1}^{N S P} v_{i j} \bar{M}_{i} \Leftrightarrow \sum_{i=1}^{N S P} v_{i j}^{\prime} \bar{M}_{i}
$$

Where $\bar{M}_{i}$ represents the $i^{\text {th }}$ chemical species name $(i=1,2, \ldots, N S P)$ and $j$ represents the $j^{\text {th }}$ reaction $(j=1$, $2, \ldots . ., L)$. These reactions proceed (forward or reverse) according to the Law of Mass Action which states: "The rate at which an elementary reaction proceeds is proportional to the product of the molar concentrations of the reactant each raised to a power equal to its stoichiometric coefficient in the reaction equation."

Let $[\bar{M}]$ denote the molar concentration of species $i$. The forward (left to right) reaction rate, Rate $_{j}(L \rightarrow R)$, for reaction $j$ can be written as:

$$
\text { Rate }_{j}(L \rightarrow R)=k_{f_{j}} \prod_{i=1}^{N S P}\left[\bar{M}_{i}\right]^{v_{i j}}
$$

Where $k_{f_{j}}$ is the forward reaction rate constant. For species $i, v_{i j}$ moles on the left side of the reaction becomes $v_{i j}^{\prime}$ moles on the right side of the reaction. Consequently, the forward reaction for reaction $j$, yields a time rate of change in the molar concentration of species $i$ as follows:

$$
(\text { Forward })=\frac{d\left[\bar{M}_{i}\right]_{j}}{d t}=\left(v_{i j}^{\prime}-v_{i j}\right) k_{f_{j}} \prod_{i=1}^{N S P}\left[\bar{M}_{i}\right]^{v_{i j}}
$$

Similarly, the reverse reaction for reaction $j$ yields:

$$
(\text { Reverse })=\frac{d\left[\bar{M}_{i}\right]_{j}}{d t}=\left(v_{i j}-v_{i j}^{\prime}\right) k_{b_{j}} \prod_{i=1}^{N S P}\left[\bar{M}_{i}\right]^{v_{i j}^{\prime}}
$$

Where $k_{b_{j}}$ is the backward reaction rate constant. Thus, the net rate of change in the molar concentration of species $i$ for reaction $j$ (denoted by $X_{i j}$ ) is as follows:

$$
X_{i j}=\frac{d\left[\bar{M}_{i}\right]_{j}}{d t}=\left(v_{i j}^{\prime}-v_{i j}\right)\left[k_{f_{j}} \prod_{i=1}^{N S P}\left[\bar{M}_{i}\right]^{v_{i j}}-k_{b_{j}} \prod_{i=1}^{N S P}\left[\bar{M}_{i}\right]^{v_{i j}^{\prime}}\right]
$$

The "species production rate" is the time rate of change for the species density. For reaction $j$ the net species production rate for species $i$ is $M_{w i} X_{i j}$ where $M_{w i}$ is the molecular weight of species $i$. Summing over all reactions gives the net species production rate $\omega_{i}$ for the reaction set.

$$
\omega_{i}=M_{w i} \sum_{j=1}^{L} X_{i j}
$$

The molar concentration of species $i$ can be written as $\frac{\rho_{i}}{M_{w i}}$, which is the species $i$ mass density divided by the species $i$ molecular weight.

$$
\left[\bar{M}_{i}\right]=\frac{\rho_{i}}{M_{w i}}=\frac{\rho C_{i}}{M_{w i}}
$$


It follows that in terms of species $i$ mass fraction Eq. (2.3.5) becomes:

$$
\begin{aligned}
& X_{i j}=\left(v_{i j}^{\prime}-v_{i j}\right)\left[k_{f_{j}} \prod_{i=1}^{N S P}\left(\frac{\rho C_{i}}{M_{w i}}\right)^{v_{i j}}-k_{b_{j}} \prod_{i=1}^{N S P}\left(\frac{\rho C_{i}}{M_{w i}}\right)^{v_{i j}^{\prime}}\right] \\
& X_{i j}=\left(v_{i j}^{\prime}-v_{i j}\right)\left[K_{j} \prod_{i=1}^{N S P}\left(\frac{\rho C_{i}}{M_{w i}}\right)^{v_{i j}}-\prod_{i=1}^{N S P}\left(\frac{\rho C_{i}}{M_{w i}}\right)^{v_{i j}^{\prime}}\right] k_{j} \\
& K_{j}=\frac{k_{f_{j}}}{k_{b_{j}}} \text { and } k_{j}=k_{b_{j}}
\end{aligned}
$$

The reaction rate $k_{j}$ is from right to left (reverse) in the above equation and is often represented by the Arrhenius form:

$$
k_{j}=a_{j} T^{-n_{j}} \exp \left(\frac{-b_{j}}{\bar{R} T}\right)
$$

Where $a_{j}$ is the pre-exponential coefficient, $n_{j}$ is the temperature dependence of the pre-exponential factor, and $b_{j}$ is the activation energy. The ratio of forward to backward rate, $K_{j}$ in Eq. (13) is related to the equilibrium constant $K_{e q l}$ by the following equation:

$$
K_{j}=\frac{k_{f_{j}}}{k_{b_{j}}}=K_{e q l}(\bar{R} T)^{\lambda_{j}}
$$

Where

$$
\lambda_{j}=\sum_{i=1}^{N S P}\left(v_{i j}^{\prime}-v_{i j}\right)
$$

The quantity $\left|\lambda_{j}\right|+1$ is known as the order of the $j^{\text {th }}$ reaction

The equilibrium constant is:

$K_{\text {eql }}=\exp \left(-\frac{\Delta F}{\bar{R} T}\right), \quad \Delta F=\sum_{i=1}^{N S P} f_{i} v_{i j}-\sum_{i=1}^{N S P} f_{i} v_{i j}^{\prime}$

$f_{i}=$ Gibbs Free Energy $=$ Chemical Potential $=\bar{h}_{i}-T \bar{S}_{i}$

$\bar{h}_{i}=$ Molar specific enthalpy

$\bar{S}_{i}=$ Molar specific entropy

Since $\bar{C}_{i}=\frac{C_{i}}{M_{w i}}($ Molar mass fraction $)$

$$
X_{i j}=\left(v_{i j}^{\prime}-v_{i j}\right)\left[K_{j} \prod_{i=1}^{N S P} \bar{C}_{i}^{v_{i j}}-\rho^{\lambda_{j}} \prod_{i=1}^{N S P} \bar{C}_{i}^{v_{i j}^{\prime}}\right] k_{j} \rho^{\sum_{i=1}^{N S P} v_{i j}}
$$




\section{RESULTS AND DISCUSSION}

Numerous test cases are compiled in References [16 and 17]. We will recite a few of these cases for this paper.

\section{$\underline{H D(M u s t a r d) \text { evaporation on non-permeable surface }}$}

Two evaporation models were developed for the use in MOCHA code. The details of these models are described in Reference [6]. Both models are compared with wind tunnel data at different temperatures, turbulence intensity, and wind speeds. Figure 1 demonstrates the results of this comparison that is quite excellent. Furthermore, the code can accept real time wind speed and temperature data and predict the evaporation based on incoming environmental information. Open air tests were conducted at Czech Republic and a sample prediction id shown in Figure 2. The real time wind speed and temperature are also embedded in this figure. The turbulence intensity was calculated from the samples that were acquired for wind velocity.

\section{Two-droplet spread and reaction on porous substrate}

The two droplet model was chosen to simulate a decontamination scenario where diffusion and chemical reaction between two different species are common. The simulation was performed with Cyclohexanol and Phosphoric acid. Equimolar droplets of cyclohexanol and phosphoric acid (cyclohexanol $89.5 \mu \mathrm{L}$, phosphoric acid $50 \mu \mathrm{L}$ ) were placed $99.5 \mathrm{~mm}$ apart on a porous media consisting of petri dishes filled with $25 \mathrm{~g}$ of glass beads, leaving $1 \mathrm{~mm}$ spacing between edges of the drops. The depth of glass beads was $0.6 \mathrm{~cm}$ and the porosity was 0.35 . The reaction produced cyclohexene when the two droplets came into contact following capillary diffusion.

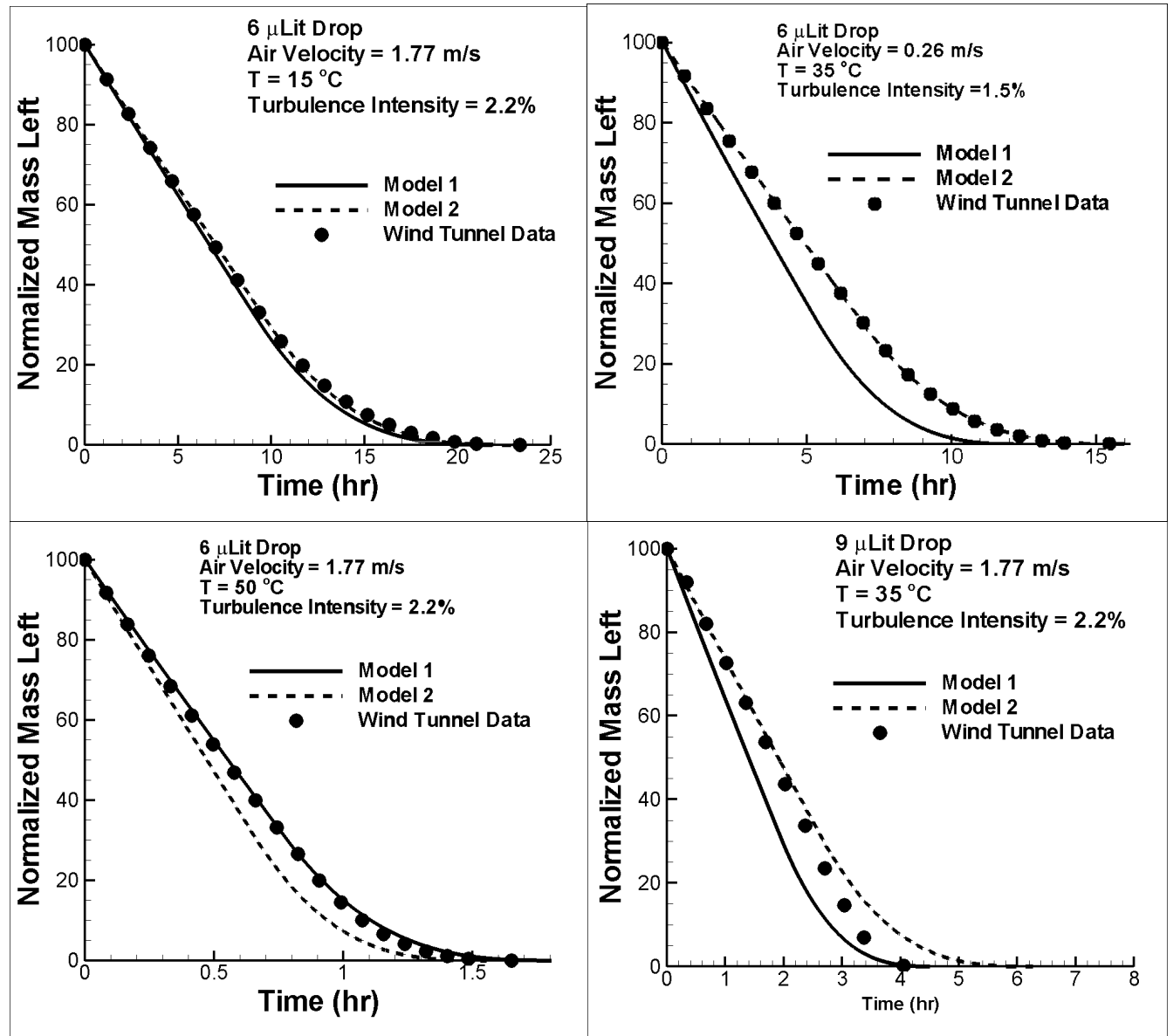

Figure 1 Single HD droplet evaporation on non-permeable surface with no chemical reaction under different environmental variables (wind speed and temperature) predicted by the MOCHA code - Comparison with wind tunnel data 


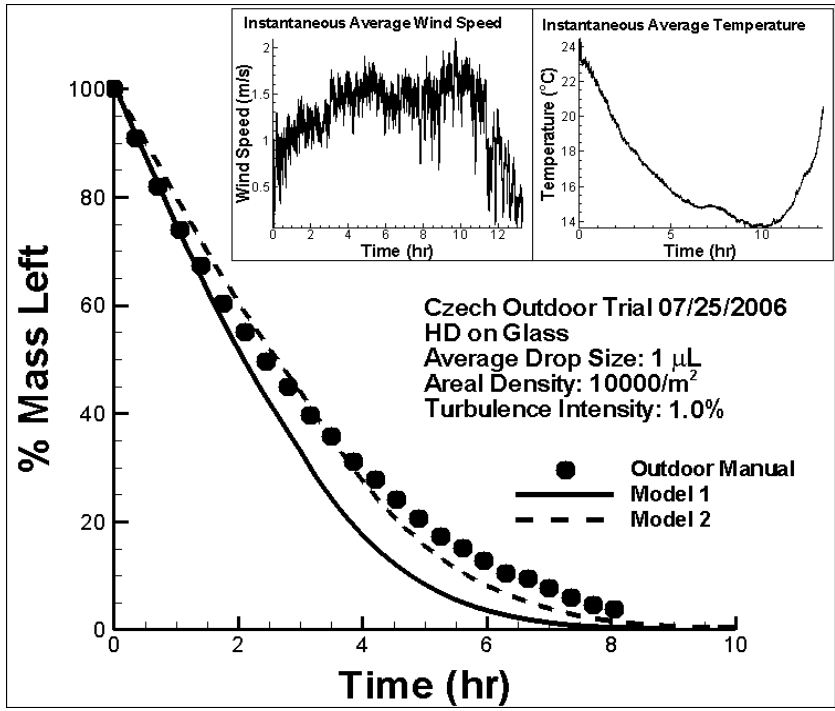

Figure 2. HD droplet evaporation on non-permeable surface with no chemical reaction under real time environmental conditions (wind speed and temperature - Model comparison with open air data

Figure 3 compares the model and experiment for the remaining Cyclohexanol. The experiment was run for about 1 hour. Figure 4 shows the contours for the mass fraction of each species and the product (Cyclohexene).

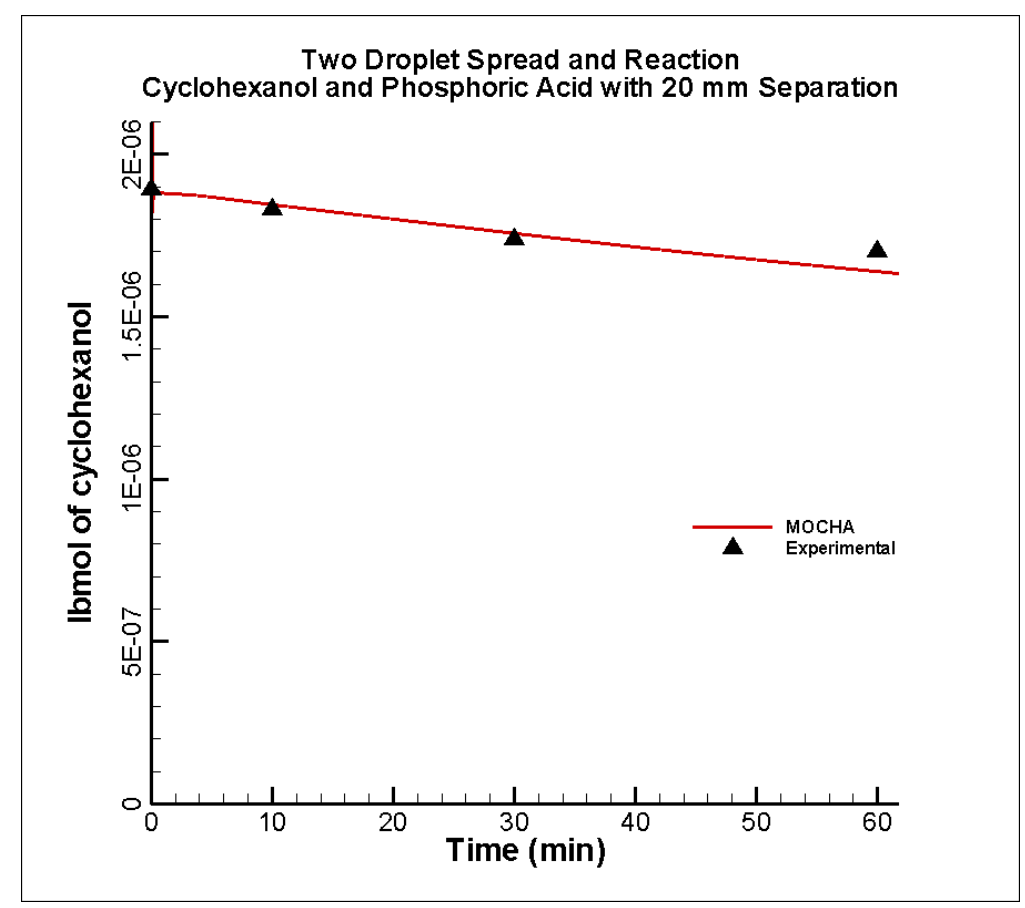

Figure 3. Simulation of a typical decontamination on a porous substrate involving chemical reaction. Phosphoric acid is used as a catalyst to turn Cyclohexanol into Cyclohexene. Model comparison with experimental data for the total moles of each species. 




Figure 4. Simulation of a typical decontamination on a porous substrate involving chemical reaction. Phosphoric acid is used as a catalyst to turn Cyclohexanol into Cyclohexene. Model prediction for species mass fraction contours.

\section{Liquid reaction with porous solid substrate}

The next test case simulates the reaction of a chemical droplet with a porous substrate. Note that for this case, the local porosity is also changing as a function of time. However, to eliminate the need for solving another differential equation for time dependent porosity, the substrate porosity is calculated at each time step and used for the next iteration, i.e. it is delayed by one time step. A $50 \mu L$ droplet of Sulfuric acid (liquid phase) was placed on a brick of sodium sulfide with the dimensions of approximately $20 \times 20 \times 15 \mathrm{~mm}^{3}$ ). The sulfuric acid mass was measured experimentally and calculated by the model. The model experiment comparison is depicted in Figure 5. 


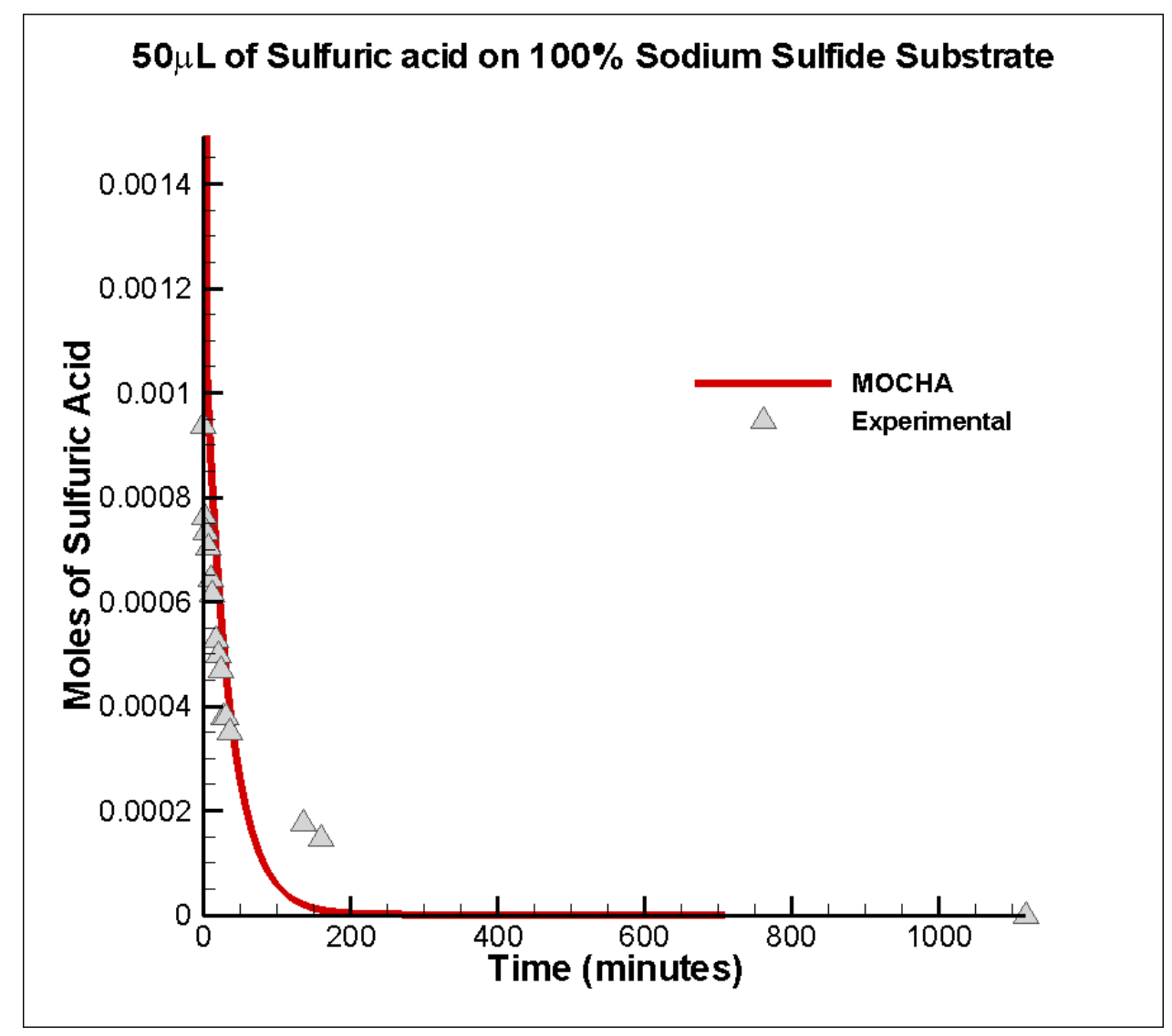

Figure 5 Remaining sulfuric acid as it reacts with sodium sulfide substrate

\section{$\underline{V X}$ hydrolysis reaction in sand}

VX nerve agent enters a complex chemical reaction with water that usually exists in soil or sand as moisture. The products of such reaction are as deadly as the VX itself. A $6 \mu \mathrm{L}$ of VX was deposited onto damp sand at $50^{\circ} \mathrm{C}$ and allowed to react. The degradation took place under sealed conditions and was therefore modeled without evaporation. The model indicated VX disappearance and appearance of breakdown products in quantities similar to those reported in the experimental study [10].

\section{CONCLUSION}

For the first time, a general purpose computational model is developed for the transport and reaction of solids, liquids, and gases within a porous substrate. The model has demonstrated to be thorough, accurate, and versatile. The comparison with experimental data somewhat indicated the robustness and applicability of the model. This MOCHA computer program can be used to study the evaporation, reaction, and transport of multi-phase species in porous (or on non-porous) substrate as frequently encountered in CHEM/BIO defense subjects. It can simulate the extent of contamination and the effectiveness of decontamination. 


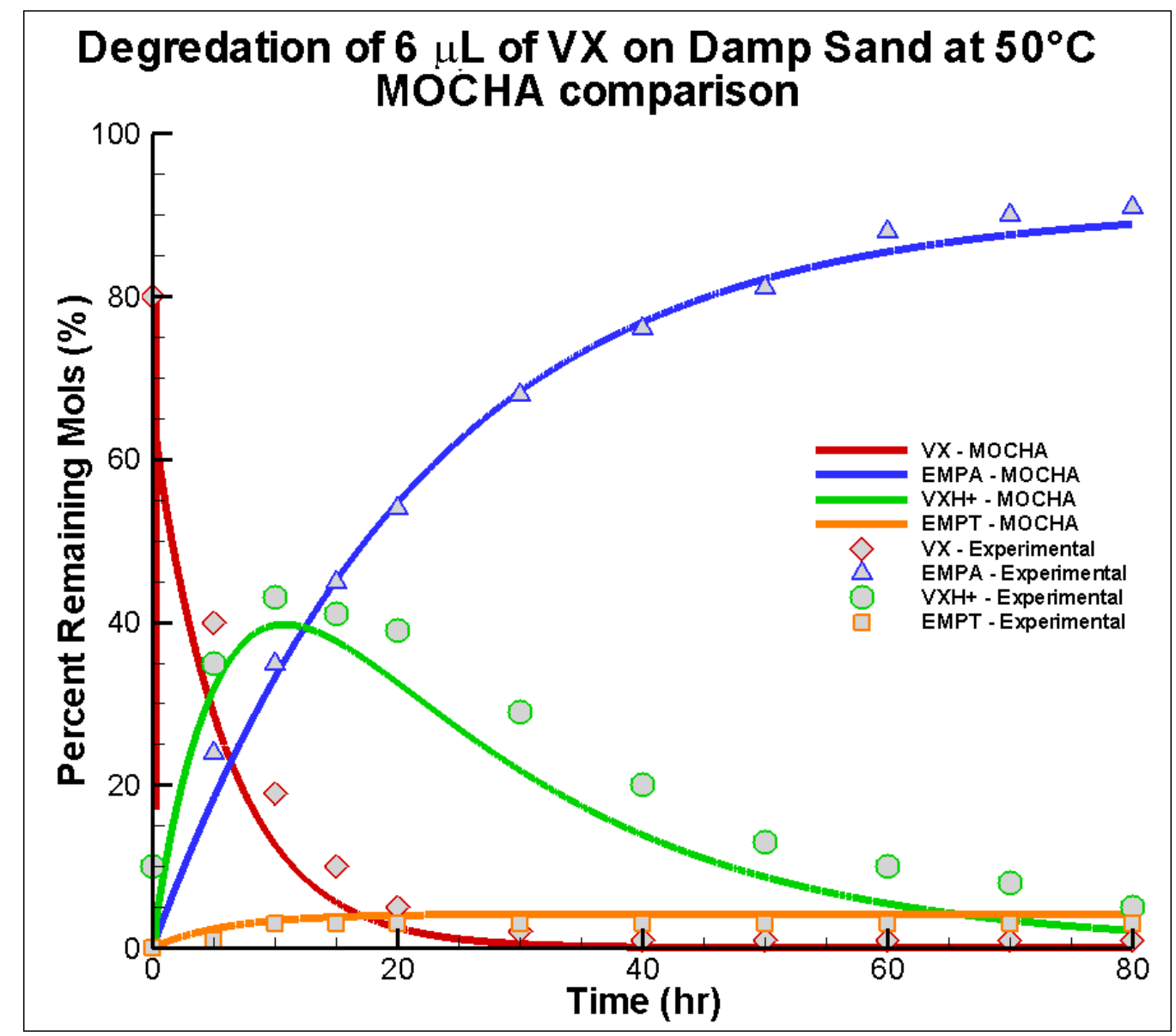

Figure 6. Percent of remaining moles for VX degradation in moist sand

\section{ACKNOWLEDGEMENTS}

This work was performed with financial supported from the Edgewood Chemical and Biological Center (ECBC) and Defense Threat Reduction Agency. 


\section{REFERENCES}

[1] B. Markicevic, H. Li, A. R. Zand and H. K. Navaz, "Types of boundary conditions in capillary secondary flow and liquid distribution," AIChE Journal. Nov. 7, 2011. Available online.

[2] B. Bijeljic, B. Markicevic and H. K. Navaz, Capillary climb dynamics in the limits of prevailing capillary and gravity force, Physical Review E, 83, Art. No. 056310, (2011).

[3] B. Markicevic, B. Bijeljic and H. K. Navaz, "Dynamics and stability of two-potential flows in the porous media," Physical Review E, Vol. 107, 2011

[4] B. Markicevic, C. Hoff, H. Li, A. R. Zand and H. K. Navaz, "Capillary force driven primary and secondary unidirectional flow of wetting liquid into porous medium," International Journal of Multiphase Flow, Vol. 39, March 2012,pp 193-204.

[5] B. Markicevic and H. K. Navaz, "The influence of capillary flow on the fate of evaporating sessile droplet imprint in the porous medium," Physics of Fluids, 22, 1 (2010).

[6] Markicevic, B., and Navaz, H.K., "Primary and secondary droplet spread into porous media," Transport in Porous Media, 85: 953-974, 2010.

[7] B. Markicevic and H. K. Navaz, The influence of capillary flow on the fate of evaporating sessile droplet imprint in the porous medium, Physics of Fluids, 22, Art. No. 122103, (2010).

[8] B. Markicevic and H. K. Navaz, "Primary and Secondary Infiltration of Wetting Droplet into Porous Media," available on-line in Transport in Porous Media, 85, 953-974, (2010).

[9] B. Markicevic, A. Nalla, H. K. Navaz, D. Heider, S. Walsh and S. G. Advani, "Closed Form Solution of Resin Flow from Multiple Line Gates in Liquid Composite Molding," Polymer Composites, 31, 1434-1441, 2010.

[10] B. Markicevic, T. G. D’Onofrio and H. K. Navaz, “On Spread Extent of Sessile Droplet into Porous Medium: Numerical Solution and Comparisons with Experiments," Physics of Fluids, 22, Art. No. 012103, 2010.

[11] T. G. D’Onofrio, B. A. Mantooth, K. B. Sumpter, B. Markicevic and H. K. Navaz, "Experimental and numerical study of spread and sorption of VX sessile droplet into medium grain-size sand," Langmuir, 26 3317 3322, 2010.

[12] B. Markicevic, H. Li, Y. Sikorski, A. R. Zand, M. Sanders and H. K. Navaz, "Infiltration Time and Imprint Shape of a Sessile Droplet Imbibing Porous Medium," Journal of Colloid and Interface Science, 336, 698-706, 2009.

[13] B. Markicevic and H. K. Navaz, "Numerical Solution of Wetting Fluid Spread into Porous Media," International Journal of Numerical Method for Heat and Fluid Flow, 19, 521-534, 2009.

[14] Markicevic, B. and Navaz, H.K., "Numerical Solution of Wetting Fluid Spread into Porous Media," International Journal of Numerical Methods for Heat and Fluid Flow, 19, 521-534, 2009.

[15] Navaz, H. K., Chan, E., and Markicevic, B., "Convective Evaporation of Sessile Droplets in a Turbulent Flow Comparison with Wind Tunnel Data," International Journal of Thermal Sciences, 47, pp. 963-971, 2008.

[16] Navaz, H. K., Zand, A., Atkinson, T., Nowakowski, A. Kiple, J., Kamensky, K., and Z. Jovic, “Agent Fate Modeling-COMCAD Theory and Analysis, Code Structure, and Users Guide, Volume I, II, and III), Final Report prepared for Edgewood Chemical and Biological Center (ECBC), January 7, 2013.

[17] Navaz, H. K., Zand, A. R., Dang, A., Gat, A., Gharib, M., Atkinson, T., Nowakowski, A., Kamensky, K., and Z. Jovic, Predictive Model for Assessment of Chemicals on and in Surfaces vs. Chemicals Available for Contact and Transport Theory and Analysis - Development of the MOCHA Computer Program, Volume I, V 2.0, Final Report Prepared for Defense Threat Reduction Agency (DTRA), March 2014. 